\title{
Hadronic decays involving heavy pentaquarks
}

\author{
Xiao-Gang $\mathrm{He}^{*}$ \\ Department of Physics, Peking University, Beijing, China \\ Xue-Qian $\mathrm{Li}^{\dagger}$ \\ Department of Physics, Nankai University, Tianjin, China \\ (Received 29 March 2004; published 31 August 2004)
}

\begin{abstract}
Recently several experiments have reported evidence for pentaquark $\Theta^{+}$. H1 experiment at HERA-B has also reported evidence for $\Theta_{c}$. $\Theta^{+}$is interpreted as a bound state of an $\bar{s}$ with four other light quarks $u d u d$ which is a member of the antidecuplet of flavor $S U(3)_{f}$, while $\Theta_{c}$ is a heavy state obtained with the $\bar{s}$ in $\Theta^{+}$ being replaced by a $\bar{c}$ which is a member of an antisixtet. A heavy pentaquark triplet can also be formed with a $\bar{c}$ and four light quarks. Similarly heavy pentaquark antisixtet and triplet can be formed with the $\bar{c}$ being replaced by a $\bar{b}$. We study decay processes involving at least one heavy pentaquark using $S U(3)_{f}$ and estimate the decay widths for some decay modes. We find several relations for heavy pentaquark decays into another heavy pentaquark and a $B\left(B^{*}\right)$ or a $D\left(D^{*}\right)$ which can be tested in the future. The $B$ can also decay through weak interaction to charmed heavy pentaquarks. We also study some $B$ decay modes with a heavy pentaquark in the final states. Experiments at $B$ factories will provide important information about the heavy pentaquark properties.
\end{abstract}

DOI: $10.1103 /$ PhysRevD.70.034030

PACS number(s): 14.20.Lq, 14.20.Mr, 14.40.Lb, 14.40.Nd

\section{INTRODUCTION}

Recently several experiments have reported evidence for pentaquarks $\Theta^{+}$and other states [1], although there are also experiments reporting null results [2]. The $\Theta^{+}(1540)$ pentaquark has strangeness $S=+1$ and has quark content $u d u d \bar{s}$. This particle is an isosinglet which is a member of the antidecuplet [3] in flavor $S U(3)_{f}$ symmetry. At present there is very limited experimental information on the detailed properties such as the decay width, spin, and parity. Several models have been proposed to accommodate these antidecuplet states [3-13].

Replacing the $\bar{s}$ in $\Theta^{+}$by a heavy quark such as $\bar{c}$ or $\bar{b}$, it is also possible to form bound heavy pentaquark states $[5,6,8,10-12,14-18] \Theta_{c}$ or $\Theta_{b}$. When implementing them into $S U(3)_{f}$, in the model of Jaffe and Wilczek [5], where pentaquark $\Theta^{+}$is composed of two $(u d)$ diquarks with spin-0 and an $\bar{s}$ quark, heavy pentaquarks form a fundamental representation of $S U(3)_{f}$ triplet $R_{c, b}$ (the subindices $c$ and $b$ indicate whether the pentaquark is formed with a $\bar{c}$ or a $\bar{b}$ ), and an antisixtet $S_{c, b}$ [10]. Discovery of these states and a study of their properties can provide important information about the inner structure of matter. H1 Collaboration has recently reported observation of a narrow resonance in Ref. [19] $D^{*-} p$ and $D^{*+} \bar{p}$ in inelastic $e P$ collisions at center-of-mass energies of 300 and $320 \mathrm{GeV}$ at HERA. This resonance has a mass of $3099 \pm 3$ (stat) \pm 5 (syst) with a Gaussian width of $12 \pm 3 \mathrm{MeV}$ and can be interpreted as evidence for $\Theta_{c}$. If this observation is confirmed, other related

\footnotetext{
* On leave of absence from Physics Department, National Taiwan University; Electronic address: hexg@phys.ntu.edu.tw

${ }^{\dagger}$ Electronic address: lixq@nankai.edu.cn
}

states should exist. In this paper we study some properties of decay processes involving at least one heavy pentaquark using $S U(3)_{f}$ flavor symmetry.

The triplet $R_{c, b}$ transforms under $S U(3)_{f}$ in a similar way as the light quark $(u, d, s)$ triplet. To indicate this fact and also to distinguish the pentaquark triplet from the quark one, we use $U, D, S$ to indicate the elements in $R_{c, b}$. For the antisixtet $S_{c, b}$, we use $T_{c, b}, N_{c, b}$, and $\Theta_{c, b}$ to indicate the isospin triplet, doublet, and singlet members, respectively. We have

$$
\begin{aligned}
& R_{c}=\left(R_{c, i}\right)=\left(U_{c}^{0}, D_{c}^{-}, S_{c}^{-}\right), \\
& S_{c}=\left(S_{c}^{i j}\right)=\left(\begin{array}{lll}
T_{c}^{--} & T_{c}^{-} / \sqrt{2} & N_{c}^{-} / \sqrt{2} \\
T_{c}^{-} / \sqrt{2} & T_{c}^{0} & N_{c}^{0} / \sqrt{2} \\
N_{c}^{-} / \sqrt{2} & N_{c}^{0} / \sqrt{2} & \Theta_{c}^{0}
\end{array}\right), \\
& R_{b}=\left(R_{b, i}\right)=\left(U_{b}^{+}, D_{b}^{0}, S_{b}^{0}\right), \\
& S_{b}=\left(S_{b}^{i j}\right)=\left(\begin{array}{lll}
T_{b}^{-} & T_{b}^{0} / \sqrt{2} & N_{b}^{0} / \sqrt{2} \\
T_{b}^{0} / \sqrt{2} & T_{b}^{+} & N_{b}^{+} / \sqrt{2} \\
N_{b}^{0} / \sqrt{2} & N_{b}^{+} / \sqrt{2} & \Theta_{b}^{+}
\end{array}\right) .
\end{aligned}
$$

The quark contents of these particles are

$$
\begin{aligned}
U_{c, b}^{0,+} & =(\text { udus })(\bar{c}, \bar{b}), \quad D_{c, b}^{-, 0}=(u d d s)(\bar{c}, \bar{b}), \\
S_{c, b}^{-, 0} & =(d s u s)(\bar{c}, \bar{b}), \\
T_{c, b}^{--,-} & =(d s d s)(\bar{c}, \bar{b}), \quad T_{c, b}^{-, 0}=(d s u s)(\bar{c}, \bar{b}), \\
T_{c, b}^{0,+} & =(\text { usus })(\bar{c}, \bar{b}),
\end{aligned}
$$




$$
\begin{aligned}
& N_{c, b}^{-, 0}=(u d d s)(\bar{c}, \bar{b}), \quad N_{c, b}^{0,+}=(u d u s)(\bar{c}, \bar{b}), \\
& \Theta_{c, b}^{0,+}=(u d u d)(\bar{c}, \bar{b}) .
\end{aligned}
$$

The $U_{c, b}, D_{c, b}$, and $N_{c, b}$ particles have $S=-1, S_{c, b}$, and $T_{c, b}$ particles have $S=-2$, and $\Theta_{c, b}$ particles have $S=0$.

In the diquark model of Ref. [5], $S_{c, b}$ have positive parity, whereas $R_{c, b}$ have negative parity since there is no $P$-wave excitation between the diquarks. In our study we emphasize on the flavor $S U(3)_{f}$ properties, the conclusions can be applied to both parity situations "+" or "- " for both $R_{c, b}$ and $S_{c, b}$.

\section{HEAVY PENTAQUARK STRONG DECAY COUPLINGS}

Whether heavy pentaquarks can have strong decay modes depends on their masses. With $S U(3)_{f}$ symmetry, particles in each multiplet are supposed to have the same mass. Quark model estimates for the masses of heavy pentaquarks have been carried out by several groups. In the diquark model, the $\Theta_{c, b}$ masses are estimated to be [5] 2710 and $6050 \mathrm{MeV}$, respectively, which are below the strong $p D$ and $n B$ decay threshold. A lattice calculation in Ref. [8], gives a $m_{\Theta}$ of about $3.5 \mathrm{GeV}$.

Removing the $P$-wave excitation energy, which is estimated by using the mass difference of $\Lambda_{c}$ and its excitation $\Lambda_{c}^{\prime}, U_{P \text {-wave }} \approx m_{\Lambda_{c}^{\prime}}-m_{\Lambda_{c}}=310 \mathrm{MeV}$, from $\Theta_{c}$, and adding a constituent strange quark contribution $\Delta_{s}=m_{\Xi_{c}}-m_{\Lambda_{c}}$ $\approx 184 \mathrm{MeV}$, Ref. [15] obtained $2580 \mathrm{MeV}$ for $U_{c}, D_{c}$ masses. Assuming the same $U_{P \text {-wave }}$ and $\Delta_{s}$ for beauty heavy pentaquarks, $U_{b}, D_{b}$ masses are estimated to be $5920 \mathrm{MeV}$ [15].

The mass degeneracy for the particles in a multiplet is lifted by quark mass differences $m_{u}, m_{d}$, and $m_{s}$. The mass terms, up to linear corrections in light quark masses, are given by

$$
\begin{aligned}
L= & m_{0}^{R_{c, b}} \operatorname{Tr}\left(\bar{R}_{c, b} R_{c, b}\right)+\alpha_{m}^{R_{c, b}} \operatorname{Tr}\left[\bar{R}_{c, b}\left(M+M^{\dagger}\right) R_{c, b}\right] \\
& +m_{0}^{S_{c, b}} \operatorname{Tr}\left(\bar{S}_{c, b} S_{c, b}\right)+\alpha_{m}^{S_{c, b}} \operatorname{Tr}\left[\bar{S}_{c, b}\left(M+M^{\dagger}\right) S_{c, b}\right]
\end{aligned}
$$

We have neglected terms of the form $\operatorname{Tr}[\bar{R}(\bar{S}) R(S)] \operatorname{Tr}(M)$ which only rescale $m_{0} . M$ is the quark mass matrix and is given by

$$
M=\left(\begin{array}{lll}
m_{u} & 0 & 0 \\
0 & m_{d} & 0 \\
0 & 0 & m_{s}
\end{array}\right)
$$

Neglecting small $m_{u, d}$ masses, we obtain

$$
\begin{aligned}
& m_{U_{c, b}}=m_{D_{c, b}}=m_{0}^{R_{c, b}}, \quad m_{S_{c, b}}=m_{0}^{R_{c, b}}+2 \alpha^{R_{c, b}} m_{s}, \\
& m_{T_{c, b}}=m_{0}^{S_{c, b}}, \quad m_{N_{c, b}}=m_{0}^{S_{c, b}}+\alpha_{m}^{S_{c, b}} m_{s}, \\
& m_{\Theta_{c, b}}=m_{0}^{S_{c, b}}+2 \alpha_{m}^{S_{c, b}} m_{s} .
\end{aligned}
$$

Taking into account the $S U(3)_{f}$ breaking effects via differences in light quark masses from $\Delta_{s}=m_{\Xi_{c}}-m_{\Lambda_{c}}$ for constituent strange quarks, the masses of $S_{c}^{-}$and $S_{b}^{0}$ were estimated to be 2770 and $6100 \mathrm{MeV}$, respectively in Ref. [15]. Making the same assumption we obtain the masses of $N_{c}$, $N_{b}, T_{c}$, and $T_{b}$ to be $2894,6236,3078$, and $6420 \mathrm{MeV}$, respectively. These values are similar to the estimates obtained in Ref. [16].

There are other model estimates for heavy pentaquark masses which give larger masses. For example, in the model of Karliner and Lipkin, where the pentaquarks are formed from a triquark and a diquark [6], the masses are estimated to be 2985 and $6398 \mathrm{MeV}$, respectively, which are above the strong $p D$ and $n B$ decay threshold. And the masses of $N_{c}$, $N_{b}, T_{c}$, and $T_{b}$ are estimated to be $3165,6570,3340$, and $6740 \mathrm{MeV}$, respectively [16]. Removing the $P$-wave excitation energy [6] $\delta E^{P \text {-wave }} \approx 207 \mathrm{MeV}$ between the diquark and triquark from $\Theta_{c, b}$ and adding the mass difference due to the replacement of a light $u$ or $d$ quark by an $s$ quark, one obtains the masses of $U_{c}^{0}, D_{c}^{-}$and $U_{b}^{+}, D_{b}^{0}$ to be 2858 and 6533 $\mathrm{MeV}$, respectively. $S_{c}^{-}$and $S_{b}^{0}$ are approximately 3028 and $6708 \mathrm{MeV}$. Clearly the above estimates for the masses are rather rough and should not be expected to hold with an accuracy better than 50 or even $100 \mathrm{MeV}$.

If the $\mathrm{H} 1$ narrow resonance of mass $3099 \mathrm{MeV}$ is indeed the $\Theta_{c}$ particle, both the diquark and triquark-diquark model predictions for the mass are slightly lower than the data. There is also the possibility that the narrow resonant state observed at $\mathrm{H} 1$ is a chiral partner of $\Theta_{c}$. At present the uncertainties involved in the estimates are large so it is too early to make a decisive conclusion. With a mass $3099 \mathrm{MeV}$ for $\Theta_{c}$, it is possible for it to decay into $D^{*-} p$ and $D^{+} \bar{p}$. Similar situation may happen for beauty pentaquarks. We therefore will consider processes involving both $D, B$ and $D^{*}, B^{*}$.

We now write down the strong decay amplitudes to the leading order using the $S U(3)_{f}$ symmetry for heavy pentaquark decays with a $B$ or $D$ in the final states. We have

$$
\begin{aligned}
& L_{R_{b} N B}=c_{R_{b} N B} \bar{R}_{b}^{j} N_{j}^{i} \bar{B}_{i}+\text { H.c., } \\
& L_{S_{b} N B}=c_{S_{b} N B} \bar{S}_{b, j k} N_{l}^{j} \bar{B}_{i} \epsilon^{i k l}+\text { H.c., } \\
& L_{R_{c} N D}=c_{R_{c} N D} \bar{R}_{c}^{j} N_{j}^{i} \bar{D}_{i}+\text { H.c., } \\
& L_{S_{c} N D}=c_{S_{c} N D} \bar{S}_{c, j k} N_{l}^{j} \bar{D}_{i} \epsilon^{i k l}+\text { H.c. }
\end{aligned}
$$

In the above $N$ is the ordinary baryon octet, $D^{i}$ and $B^{i}$ are the charm and beauty mesons. They are given by 
TABLE I. Couplings for $B \bar{N} R_{b}\left(D \bar{N} R_{c}\right)$ in unit $c_{R N B}\left(c_{R N D}\right)$. The Lorentz structure for the bi-spinor product is of the form $\overline{N \Gamma}_{P} R$ with $\Gamma_{P}=+1$ and $\gamma_{5}$ for negative and positive parity for $R$, respectively. For $B^{*} \bar{N} R_{b}$, the Lorentz structure for the bispinor product should be changed to $\bar{N} \gamma_{\mu} \gamma_{5} \Gamma_{P} R_{b}$.

\begin{tabular}{lccc}
\hline \hline$B_{u}$ & $\left(\frac{1}{\sqrt{6}} \bar{\Lambda}+\frac{1}{2} \Sigma^{0}\right) U_{b}^{+}+\Sigma^{-} D_{b}^{0}+\Xi^{-} S_{b}^{0}$ & $D_{u}$ & $\left(\frac{1}{\sqrt{6}} \bar{\Lambda}+\frac{1}{2} \Sigma^{0}\right) U_{c}^{0}+\Sigma^{-} D_{c}^{-}+\Xi^{-} S_{c}^{-}$ \\
$B_{d}$ & $\Sigma^{+} U_{b}^{+}+\left(\frac{1}{\sqrt{6}} \bar{\Lambda}-\frac{1}{\sqrt{2}} \bar{\Sigma}^{0}\right) D_{b}^{0}+\bar{\Xi}^{0} S_{b}^{0}$ & $D_{d}$ & $\bar{\Sigma}^{+} U_{c}^{0}+\left(\frac{1}{\sqrt{6}} \bar{\Lambda}-\frac{1}{\sqrt{2}} \bar{\Sigma}^{0}\right) D_{c}^{-}+\Xi^{0} S_{c}^{-}$ \\
$B_{s}$ & $\bar{p} U_{b}^{+}+\bar{n} D_{b}^{0}-\sqrt{\frac{2}{3}} \bar{\Lambda} S_{b}^{0}$ & $D_{s}$ & $\bar{p} U_{c}^{0}+\bar{n} D_{c}^{-}-\sqrt{\frac{2}{3}} \bar{\Lambda} S_{c}^{-}$ \\
\hline \hline
\end{tabular}

$$
\begin{aligned}
& N=\left(N_{i}^{j}\right)=\left(\begin{array}{lll}
\frac{\Sigma^{0}}{\sqrt{2}}+\frac{\Lambda}{\sqrt{6}} & \Sigma^{+} & p \\
\Sigma^{-} & -\frac{\Sigma^{0}}{\sqrt{2}}+\frac{\Lambda}{\sqrt{6}} & n \\
\Xi^{-} & \Xi^{0} & -\frac{2 \Lambda}{\sqrt{6}}
\end{array} \mid .\right. \\
& B=\left(B^{i}\right)=\left(B_{u}, B_{d}, B_{s}\right)=(b \bar{u}, b \bar{d}, b \bar{s}) .
\end{aligned}
$$

In Tables I and II, we list the couplings for $\bar{N} R_{b}\left(S_{b}\right) B$. One can obtain the couplings for $\bar{N} R_{c}\left(S_{c}\right) D$ by replacing $B_{i}$ by $D_{i}$ and the subindex $b$ to $c$. Throughout the paper, in all the equations for pentaquark couplings only group indices are properly labeled and all fields in the Lagrangian are going outwards. The Lorentz structures are suppressed in the equations, while the proper ones are given in the tables. Also we will assume that heavy pentaquarks are spin-1/2 particles. To obtain results for spin-3/2 heavy pentaquarks, one can just use at an appropriate place the Rarita-Schwinger vector spinor for the relevant fields.

We would like to point out that the above equations can be equally applied to processes with $B$ and $D$ replaced by $D^{*}$

\begin{tabular}{|c|c|}
\hline$B_{u}$ & $\begin{array}{l}\frac{1}{2}\left[\sqrt{2} \Xi^{-} T_{b}^{0}+2 \Xi^{0} T_{b}^{+}-\sqrt{2} \bar{\Sigma}^{-} N_{b}^{0}-\sqrt{3} \bar{\Lambda} N_{b}^{+}+\bar{\Sigma}^{0} N_{b}^{+}\right. \\
\left.\quad-2 \bar{n} \Theta_{b}^{+}\right]\end{array}$ \\
\hline$B_{d}$ & $\begin{array}{l}\frac{1}{2}\left[-2 \Xi^{-} T_{b}^{-}-\sqrt{2} \Xi^{0} T_{b}^{0}+\sqrt{2} \bar{\Sigma}^{+} N_{b}^{+}+\sqrt{3} \bar{\Lambda} N_{b}^{0}+\bar{\Sigma}^{0} N_{b}^{0}\right. \\
\left.\quad+2 \bar{p} \Theta_{b}^{+}\right]\end{array}$ \\
\hline$B_{s}$ & $\frac{1}{2}\left[2 \bar{\Sigma}^{-} T_{b}^{-}-2 \bar{\Sigma}^{0} T_{b}^{0}-2 \Sigma^{+} T_{b}^{+}-\sqrt{2} \bar{p} N_{b}^{+}+\sqrt{2} \bar{n} N_{b}^{0}\right]$ \\
\hline$D_{u}$ & $\begin{array}{l}\frac{1}{2}\left[\sqrt{2} \Xi^{-} T_{c}^{-}+2 \Xi^{0} T_{c}^{0}-\sqrt{2} \Sigma^{-} N_{c}^{-}-\sqrt{3} \bar{\Lambda} N_{c}^{0}+\bar{\Sigma}^{0} N_{c}^{0}\right. \\
\left.\quad-2 \bar{n} \Theta_{c}^{0}\right]\end{array}$ \\
\hline$D_{d}$ & $\begin{array}{l}\frac{1}{2}\left[-2 \Xi^{-} T_{c}^{--}-\sqrt{2} \Xi^{0} T_{c}^{-}+\sqrt{2} \Sigma^{+} N_{c}^{0}+\sqrt{3} \bar{\Lambda} N_{c}^{-}\right. \\
+\Sigma^{0} N_{c}^{-} \\
\left.\quad+2 \bar{p} \Theta_{c}^{0}\right]\end{array}$ \\
\hline$D_{s}$ & $\frac{1}{2}\left[2 \Sigma^{-} T_{c}^{--}-2 \Sigma^{0} T_{c}^{-}-2 \Sigma^{+} T_{c}^{0}-\sqrt{2} \bar{p} N_{c}^{0}+\sqrt{2} \bar{n} N_{c}^{-}\right]$ \\
\hline
\end{tabular}

TABLE II. Couplings for $B \bar{N} S_{b}\left(D \bar{N} S_{c}\right)$ in unit $c_{S N B}\left(c_{S N D}\right)$. The Lorentz structure is the same as in Table I. and $B^{*}$, respectively. We will not distinguish them in the equations in later discussions unless specifically indicated.

If the diquark model for pentaquarks is the right one, we see that all the strong decay modes are forbidden due to restriction of phase space. However, if the masses are close to the triquark-diquark model predictions, strong decays are allowed. The $\mathrm{H} 1$ data indicate that the above strong decays are possible. One can use the allowed decay modes to determine the parameter $c_{a b c}$ and then the widths of the heavy pentaquarks. Here we give the formula for the couplings in terms of decay widths assuming the decays are allowed. For decays with $B$ in the final states we have

$$
\begin{aligned}
c_{R_{b} N B}^{2} & =\frac{16 \pi m_{S_{b}^{0}} \Gamma\left(S_{b}^{0} \rightarrow \Xi^{-} \bar{B}_{u}\right)}{\left[\left(\hat{\pi} m_{S_{b}^{0}}+m_{\Xi^{-}}\right)^{2}-m_{B_{u}}^{2}\right] P h\left(m_{S_{b}^{0}}, m_{\Xi^{-}}, m_{B_{u}}\right)}, \\
c_{S_{b} N B}^{2} & =\frac{16 \pi m_{\Theta_{b}^{+}} \Gamma\left(\Theta_{b}^{+} \rightarrow p \bar{B}_{d}\right)}{\left[\left(\hat{\pi} m_{\Theta_{b}} m_{p}\right)^{2}-m_{B_{d}}^{2}\right] P h\left(m_{\Theta_{b}^{+}}, m_{p}, m_{B_{d}}\right)},
\end{aligned}
$$

where $P h(a, b, c)=\sqrt{\left.1-(b+c)^{2} / a^{2}\right)\left(1-(b-c)^{2} / a^{2}\right)} . \hat{\pi}$ is the eigenvalue of parity of the heavy pentaquark.

For decays with $B^{*}$ in the final states, we have

$$
\begin{aligned}
c_{R_{b} N B^{*}}^{2} & =\frac{16 \pi m_{S_{b}^{0}} \Gamma\left(S_{b}^{0} \rightarrow \Xi^{-} \bar{B}_{u}^{*}\right)}{f\left(m_{S_{b}^{0}, m_{\Xi}}, m_{B_{u}^{*}}\right) P h\left(m_{S_{b}^{0}}, m_{\Xi^{-}}, m_{B_{u}^{*}}\right)}, \\
c_{S_{b} N B^{*}}^{2} & =\frac{16 \pi m_{\Theta_{b}^{+}} \Gamma\left(\Theta_{b}^{+} \rightarrow p \bar{B}_{d}^{*}\right)}{f\left(m_{\Theta_{b}^{+}}, m_{p}, m_{B_{d}^{*}}\right) P h\left(m_{\Theta_{b}^{+}}, m_{p}, m_{B_{d}^{*}}\right)},
\end{aligned}
$$

where

$$
f(a, b, c)=a^{2}+b^{2}-c^{2}-\hat{\pi} 6 a b+\left[\left(a^{2}-b^{2}\right)^{2}-c^{4}\right] / c^{2} .
$$

Similarly one can obtain $c_{R_{c}\left(S_{c}\right) N D\left(D^{*}\right)}^{2}$ by considering $S_{c}^{-} \rightarrow \bar{D}_{u}^{0}\left(D_{u}^{* 0}\right) \Xi^{-}$and $\Theta_{c}^{0} \rightarrow p \bar{D}_{d}^{+}\left(\bar{D}_{d}^{*+}\right)$ decays, respectively. At present there is only some information on the width of $\Gamma\left(\Theta_{c} \rightarrow p \bar{D}_{d}^{*+}\right)$. Assuming that the narrow resonant state of width $12 \pm 3 \mathrm{MeV}$ at $\mathrm{H} 1$ is the $\Theta_{c}$ particle, we obtain

$$
c_{S_{c} N D^{*}}^{2} \approx \begin{cases}1.712, & \hat{\pi}=1, \\ 0.167, & \hat{\pi}=-1 .\end{cases}
$$


Using these numbers, the decay widths for decay modes in Table II involving $D^{*}$ can be predicted. These predictions can be tested.

\section{WEAK HADRONIC DECAYS OF HEAVY PENTAQUARKS}

Heavy pentaquark can also decay through weak interaction. If kinematically the strong decays discussed in the previous section are not allowed, weak interaction will dominate heavy pentaquark decays. These decays can be semileptonic or purely hadronic ones. Analysis on some of the heavy pentaquark properties have been carried out $[11,12,15-18]$. Here we will concentrate on some two body hadronic heavy pentaquark decays.

\section{A. $R_{b}\left(S_{b}\right) \rightarrow R_{c}\left(S_{c}\right)+\Pi$ decays}

In this subsection we study pentaquark decays of the type $R_{b}\left(S_{b}\right) \rightarrow R_{c}\left(S_{c}\right) \Pi$. Here $\Pi$ represents a meson in the pesudoscalar octet which is given by

$$
\left.\Pi=\left(\Pi_{i}^{j}\right)=\mid \begin{array}{lll}
\frac{\pi^{0}}{\sqrt{2}}+\frac{\eta}{\sqrt{6}} & \pi^{+} & K^{+} \\
\pi^{-} & -\frac{\pi^{0}}{\sqrt{2}}+\frac{\eta}{\sqrt{6}} & K^{0} \\
K^{-} & \bar{K}^{0} & -\frac{2 \eta}{\sqrt{6}}
\end{array}\right) .
$$

The quark level effective Hamiltonian for $R_{b}\left(S_{b}\right)$ $\rightarrow R_{c}\left(S_{c}\right) \Pi$ is given by

$$
\begin{aligned}
H_{\mathrm{eff}}= & \frac{G_{F}}{\sqrt{2}}\left[V_{c b}^{*} V_{u q}\left(c_{1} O_{1}+c_{2} O_{2}\right)\right. \\
& \left.+V_{u b} V_{c q}^{*}\left(c_{1} \widetilde{O}_{1}+c_{2} \widetilde{O}_{2}\right)\right], \\
O_{1}= & \bar{b} \gamma_{\mu}\left(1-\gamma_{5}\right) c \bar{u} \gamma^{\mu}\left(1-\gamma_{5}\right) q, \\
O_{2}= & \bar{b} \gamma_{\mu}\left(1-\gamma_{5}\right) q \bar{u} \gamma^{\mu}\left(1-\gamma_{5}\right) c, \\
\widetilde{O}_{1}= & \bar{b} \gamma_{\mu}\left(1-\gamma_{5}\right) u \bar{c} \gamma^{\mu}\left(1-\gamma_{5}\right) q, \\
\widetilde{O}_{2}= & \bar{b} \gamma_{\mu}\left(1-\gamma_{5}\right) q \bar{c} \gamma^{\mu}\left(1-\gamma_{5}\right) u .
\end{aligned}
$$

The two operators $O_{1,2}$ in the above can induce decays of the type $R_{b}\left(S_{b}\right) \rightarrow R_{c}\left(S_{c}\right)+\Pi$, while the operators $\widetilde{O}_{1,2}$ will not cause beauty heavy pentaquark to charmed pentaquark transitions. We write it down here for later discussions.

Under the $S U(3)_{f}$ symmetry $O_{1,2}$ transforms as an octet $H_{j}^{i}$. With proper normalization the nonzero entries of $H_{j}^{i}$ can be written as

$$
q=d, \quad H_{2}^{1}=1 ; \quad q=s, \quad H_{3}^{1}=1 .
$$

The $S U(3)_{f}$ invariant decay amplitudes are

$$
\begin{aligned}
H\left(R_{b} \rightarrow R_{c} \Pi\right)= & V_{c b}^{*} V_{u q}\left[r_{81} \bar{R}_{b, i} R_{c}^{i} \Pi_{k}^{j} H_{j}^{k}+r_{82} \bar{R}_{b i} R_{c}^{j} \Pi_{j}^{k} H_{k}^{i}\right. \\
& \left.+r_{83} \bar{R}_{b, i} R_{c}^{j} \Pi_{k}^{i} H_{j}^{k}\right], \\
H\left(R_{b} \rightarrow S_{c} \Pi\right)= & V_{c b}^{*} V_{u q}\left[s_{81} \bar{R}_{b i} S_{c, j l} \Pi_{k}^{m} H_{m}^{l} \epsilon^{i j k}+s_{82} \bar{R}_{b i} S_{c, j l}\right. \\
& \left.\times \Pi_{m}^{i} H_{k}^{l} \epsilon^{j k m}+s_{83} R_{b, i} \bar{S}_{c, j l} \Pi_{m}^{l} H_{k}^{i} \epsilon^{j k m}\right] .
\end{aligned}
$$

The amplitudes for $S_{b} \rightarrow R_{c} \Pi$ can be obtained by interchanging the indices $b$ and $c$, and treating the processes as the charge conjugated ones in the second equation of Eq. (16).

The amplitudes for $S_{b} \rightarrow S_{c} \Pi$ can be written as

$$
\begin{aligned}
H\left(S_{b} \rightarrow S_{c} \Pi\right)= & V_{c b}^{*} V_{u q}\left[s_{1} \bar{S}_{b}^{i j} S_{c, i j} \Pi_{k}^{l} H_{l}^{k}+s_{2} \bar{S}_{b}^{i j} S_{c, k l} \Pi_{k}^{i} H_{l}^{j}\right. \\
& \left.+s_{3} \bar{S}_{b}^{i j} S_{c, k j} \Pi_{k}^{l} H_{l}^{i}+s_{4} \bar{S}_{b}^{i j} S_{c, k j} \Pi_{l}^{i} H_{k}^{l}\right] .
\end{aligned}
$$

We list the results in Tables III, IV, and V. One can easily generalize the above formulation to the case with the vector meson nonet $\left(\rho^{0, \pm}, K^{*, 0, \pm}, \omega, \phi\right)$.

\section{B. $\boldsymbol{R}_{b}\left(S_{b}\right) \rightarrow R_{c}\left(S_{c}\right) D\left(D^{*}\right)$}

The effective Hamiltonian for these processes is given by

$$
H_{\mathrm{eff}}=\frac{G_{F}}{\sqrt{2}} V_{c b}^{*} V_{c q}\left(c_{1} O_{1}^{c}+c_{2} O_{2}^{c}\right)
$$

Here $O_{1}^{c}=\overline{b \gamma}^{\mu}\left(1-\gamma_{5}\right) c \bar{c}_{\mu}\left(1-\gamma_{5}\right) q \quad$ and $\quad O_{2}^{c}=\overline{c \gamma}^{\mu}(1$ $\left.-\gamma_{5}\right) c \overline{b \gamma}_{\mu}\left(1-\gamma_{5}\right) q$. In the above we have neglected small penguin contributions. This effective Hamiltonian transforms as a triplet 3 with nonzero entries

$$
q=d, H_{2}=1 ; q=s, H_{3}=1 .
$$

The $S U(3)_{f}$ invariant amplitudes can be written as

$$
\begin{aligned}
H\left[R_{b}\left(S_{b}\right) \rightarrow\right. & \left.R_{c}\left(S_{c}\right) D\right] \\
= & V_{c b}^{*} V_{c q}\left[r_{31} \bar{R}_{b}^{i} R_{c, i} H_{j} D^{j}\right. \\
& +r_{32} \bar{R}_{b}^{i} H_{i} R_{c, j} D^{j}+r \epsilon_{i j k} \bar{R}_{b}^{i} S^{c, j l} D^{k} H_{l} \\
& \left.+s_{31} \bar{S}_{b, i j} S_{c}^{i j} H_{k} D^{k}+s_{32} \bar{S}_{b, i j} S^{i k} H_{k} D^{j}\right] .
\end{aligned}
$$

The results for individual processes are given in Table VI.

$$
\text { C. } B \rightarrow N \bar{R}_{c}\left(\bar{S}_{c}\right) \text { and } B \rightarrow \bar{N} R_{c}\left(S_{c}\right)
$$

The conjugate operators of $O_{1,2}$ in Eq. (13) are of the form $\overline{c b} \overline{q u}$ and can induce decays of the type $B \rightarrow N$ $+\bar{R}_{c}\left(\bar{S}_{c}\right)$. The $S U(3)_{f}$ invariant decay amplitudes are

$$
\begin{aligned}
H\left(R_{c}\right)= & V_{c b} V_{u q}^{*}\left[\tilde{r}_{81} \bar{B}_{i} \bar{R}_{c}^{j} N_{k}^{i} H_{j}^{k}+\tilde{r}_{82} \bar{B}_{i} \bar{R}_{c}^{j} N_{j}^{k} H_{k}^{i}\right. \\
& \left.+\tilde{r}_{83} \bar{B}_{i} \bar{R}_{c}^{i} N_{k}^{j} H_{j}^{k}\right], \\
H\left(S_{c}\right)= & V_{c b} V_{u q}^{*}\left[\tilde{s}_{81} \bar{B}_{i} \bar{S}_{c j l} N_{k}^{m} H_{m}^{l} \epsilon^{i j k}\right. \\
& \left.+\tilde{s}_{82} \bar{B}_{i} \bar{S}_{c j l} N_{m}^{i} H_{k}^{l} \epsilon^{j k m}+\tilde{s}_{83} B_{i} \bar{S}_{c j l} N_{m}^{l} H_{k}^{i} \epsilon^{j k m}\right] .
\end{aligned}
$$


TABLE III. $S U(3)$ decay amplitudes for $R_{b} \rightarrow R_{c}\left(S_{c}\right) \Pi$. The Lorentz structure of the bispinor product is of the form $\bar{R}_{c}\left(\bar{S}_{c}\right)$ (1 $\left.+b \gamma_{5}\right) R_{b}$. Here $b$ is a parameter.

\begin{tabular}{|c|c|c|c|}
\hline$\overline{U_{b}^{+}}$decay & $\Delta S=0$ & & $\Delta S=1$ \\
\hline$\overline{U_{c}^{0} \pi^{+}}$ & $r_{81}+r_{83}$ & $U_{c}^{0} K^{+}$ & $r_{81}+r_{83}$ \\
\hline$T_{c}^{0} K^{+}$ & $s_{81}-s_{82}$ & $N_{c}^{0} K^{+}$ & $\frac{1}{\sqrt{2}}\left(s_{81}-s_{82}\right)$ \\
\hline$N_{c}^{0} \pi^{+}$ & $-\frac{1}{\sqrt{2}}\left(s_{81}-s_{82}\right)$ & $\Theta_{c}^{0} \pi^{+}$ & $-\left(s_{81}-s_{82}\right)$ \\
\hline \multicolumn{4}{|l|}{$D_{b}^{0}$ decay } \\
\hline$U_{c}^{0} K^{0}$ & $\frac{1}{\sqrt{2}}\left(r_{82}-r_{83}\right)$ & $U_{c}^{0} \pi^{0}$ & $r_{83}$ \\
\hline$U_{c}^{0} \eta$ & $\frac{1}{\sqrt{6}}\left(r_{82}+r_{83}\right)$ & $D_{c}^{-} K^{+}$ & $r_{81}$ \\
\hline$D_{c}^{-} \pi^{+}$ & $r_{81}+r_{82}$ & $N_{c}^{-} K^{+}$ & $-\frac{1}{\sqrt{2}} s_{81}$ \\
\hline$S_{c}^{-} K^{+}$ & $r_{82}$ & $N_{c}^{0} K^{0}$ & $-\frac{1}{\sqrt{2}} s_{82}$ \\
\hline$T_{c}^{-} K^{+}$ & $-\frac{1}{\sqrt{2}}\left(s_{81}+s_{83}\right)$ & $\Theta_{c}^{0} \pi^{0}$ & $\frac{1}{\sqrt{2}}\left(s_{81}-s_{82}\right)$ \\
\hline$T_{c}^{0} K^{0}$ & $-\left(s_{82}+s_{83}\right)$ & $\Theta_{c}^{0} \eta$ & $\frac{1}{\sqrt{6}}\left(s_{81}+s_{82}\right)$ \\
\hline$N_{c}^{-} \pi^{+}$ & $\frac{1}{\sqrt{2}} s_{83}$ & & \\
\hline$N_{c}^{0} \pi^{0}$ & $\frac{1}{2}\left(s_{81}-s_{82}-s_{83}\right)$ & & \\
\hline$N_{c}^{0} \eta$ & $\frac{1}{2 \sqrt{3}}\left(s_{81}+s_{82}+3 s_{83}\right)$ & & \\
\hline$\Theta_{c}^{0} \bar{K}^{0}$ & $s_{83}$ & & \\
\hline \multicolumn{4}{|l|}{$S_{b}^{0}$ decay } \\
\hline$U_{c}^{0} \bar{K}^{0}$ & $r_{83}$ & $U_{c}^{0} \pi^{0}$ & $\frac{1}{\sqrt{2}} r_{82}$ \\
\hline$S_{c}^{-} \pi^{+}$ & $r_{81}$ & $U_{c}^{0} \eta$ & $\frac{1}{\sqrt{6}}\left(2 r_{82}-r_{83}\right)$ \\
\hline$T_{c}^{-} \pi^{+}$ & $\frac{1}{\sqrt{2}} s_{81}$ & $D_{c}^{-} \pi^{+}$ & $r_{82}$ \\
\hline$T_{c}^{0} \pi^{0}$ & $-\frac{1}{\sqrt{2}} s_{81}$ & $S_{c}^{-} K^{+}$ & $r_{81}+r_{82}$ \\
\hline$T_{c}^{0} \eta$ & $-\frac{1}{\sqrt{6}}\left(s_{81}-2 s_{82}\right)$ & $T_{c}^{-} K^{+}$ & $-\frac{1}{\sqrt{2}} s_{83}$ \\
\hline \multirow[t]{5}{*}{$N_{c}^{0} \bar{K}^{0}$} & $\frac{1}{\sqrt{2}} s_{82}$ & $T_{c}^{0} K^{0}$ & $-s_{83}$ \\
\hline & & $N_{c}^{-} \pi^{+}$ & $\frac{1}{\sqrt{2}}\left(s_{81}+s_{83}\right)$ \\
\hline & & $N_{c}^{0} \pi^{0}$ & $-\frac{1}{2}\left(s_{81}+s_{83}\right)$ \\
\hline & & $N_{c}^{0} \eta$ & $-\frac{1}{2 \sqrt{3}}\left(s_{81}-2 s_{82}-3 s_{83}\right)$ \\
\hline & & $\Theta_{c}^{0} \bar{K}^{0}$ & $s_{82}+s_{83}$ \\
\hline
\end{tabular}

The conjugate operators $\widetilde{O}_{1,2}$ in Eq. (14) are of the form $\bar{q} b \bar{u} c$. These operators can induce $B \rightarrow \bar{N}+R_{c}\left(S_{c}\right)$. It contains an $S U(3)$ triplet and an antisixtet. The nonzero entries are

$$
\begin{aligned}
& q=d, \quad H(3 c)_{3}=1, \quad H(6 c)^{12}=H(6 c)^{21}=1 ; \\
& q=s, \quad H(3 c)_{2}=-1, \quad H(6 c)^{13}=H(6 c)^{31}=1 .
\end{aligned}
$$

One can write down $S U(3)_{f}$ decay amplitudes for $B$ $\rightarrow \bar{N} R_{c}\left(S_{c}\right)$ as

$$
\begin{aligned}
H\left(R_{c}\right)= & V_{u b} V_{c q}^{*}\left[\tilde{r}_{31} \bar{B}_{i} R_{c j} \bar{N}_{l}^{i} H_{k} \epsilon^{j l k}+\tilde{r}_{32} \bar{B}_{i} R_{c j} \bar{N}_{l}^{j} H_{k} \epsilon^{i l k}\right. \\
& \left.+\tilde{r}_{61} \bar{B}_{i} R_{c j} \bar{N}_{k}^{i} H^{j k}+\tilde{r}_{62} \bar{B}_{i} R_{c j} \bar{N}_{k}^{j} H^{i k}\right] \\
H\left(S_{c}\right)= & V_{u b} V_{c q}^{*}\left[\tilde{s}_{31} \bar{B}_{i} S_{c}^{i j} \bar{N}_{j}^{k} H_{k}+\tilde{s}_{32} \bar{B}_{i} S_{c}^{j k} \bar{N}_{j}^{i} H_{k}\right. \\
& \left.+\tilde{s}_{61} \bar{B}_{i} S_{c}^{i j} \bar{N}_{l}^{k} H^{l m} \epsilon_{j k m}+\tilde{s}_{62} \bar{B}_{i} S_{c}^{j k} \bar{N}_{k}^{l} H^{i m} \epsilon_{j l m}\right] .
\end{aligned}
$$

The hadronic parameters $\tilde{r}_{i j}$ are expected to be similar, $\Gamma\left[B \rightarrow \bar{N} R_{c}\left(S_{c}\right)\right]$ would be smaller by a factor of $\left|V_{c b} V_{u q}^{*}\right|^{2} /\left|V_{u b} V_{c q}^{*}\right|^{2}$ compared with $\Gamma\left(B \rightarrow N \bar{R}_{c},\left(\bar{S}_{c}\right)\right)$. The details are listed in Tables VII, VIII, and IX.

\section{DISCUSSIONS AND CONCLUSIONS}

If the recently discovered state $\Theta^{+}$is interpreted as a pentaquark bound state with an $\bar{s}$ and four light quarks, heavy pentaquarks with the $\bar{s}$ replaced by a $\bar{b}$ or a $\bar{c}$ should exist. H1 experiment at HERA-B has obtained some evidence for $\Theta_{c}$. These states form $S U(3)_{f}$ triplets $R_{c, b}$ and antisixtets $S_{c, b} \cdot R_{c}$ and $S_{c}$ can also be produced from $B$ decays at $B$ factories. If pentaquarks $R_{c, b}$ and $S_{c, b}$ are kinematically allowed to decay through strong interactions, one can use Tables I and II to relate different decay widths, and to test the model.

At present there is only some evidence for $\Theta_{c} \rightarrow D^{*-} p$. Using the decay width $\Gamma=12 \mathrm{MeV}$ obtained from $\mathrm{H} 1$, we determine $c_{S N D *}^{2}$ to be 1.712 and 0.167 for $\Theta_{c}$ with positive and negative parities, respectively. One expects $c_{S N B *}^{2}$ to be similar to $c_{S N D^{*}}^{2}$. Using Table II, we can obtain other decay widths which can be tested in the future. We have nothing much to say about the size of the couplings except that we expect the couplings $c_{R_{b} N B}$ and $c_{S_{b} N D}$ to be about the same as $c_{R_{c} N D}$ and $c_{S_{c} N D}$, respectively. If one extends $S U(3)_{f}$ to $S U(4)_{f}$, the strong couplings can be related in principle to the ones involving just light pentaquarks [12]. We will not consider this possibility here.

We have parametrized some of the two body hadronic weak decays of heavy pentaquarks in terms of $S U(3)_{f}$ invariant amplitudes. From the tables obtained we see that there are several relations among different decay modes. For example, for $\Delta S=0$ processes of the type $S_{b} \rightarrow S_{c} \Pi$, from Table $\mathrm{V}$ we obtain 
TABLE IV. $S U(3)$ decay amplitudes for $S_{b} \rightarrow S_{c} \Pi$ with $\Delta S=0$. The Lorentz structure is similar to Table III.

\begin{tabular}{|c|c|c|c|c|c|c|}
\hline$T_{b}^{-}$ & $T_{c}^{--} \pi^{+}$ & $T_{c}^{-} \pi^{0}$ & $T_{c}^{-} \eta$ & $T_{c}^{0} \pi^{-}$ & $N_{c}^{-} \bar{K}^{0}$ & $N_{c}^{0} K^{-}$ \\
\hline & $s_{1}+s_{3}$ & $\frac{1}{2}\left(s_{2}-s_{3}+s_{4}\right)$ & $\frac{1}{2 \sqrt{3}}\left(s_{2}+s_{3}+s_{4}\right)$ & $s_{2}$ & $\frac{1}{\sqrt{2}} s_{3}$ & $\frac{1}{\sqrt{2}} s_{2}$ \\
\hline \multirow[t]{2}{*}{$T_{b}^{0}$} & $T_{c}^{-} \pi^{+}$ & $T_{c}^{0} \pi^{0}$ & $T_{c}^{0} \eta$ & $N_{c}^{0} \bar{K}^{0}$ & & \\
\hline & $\frac{1}{2}\left(s_{1}+s_{2}+s_{4}\right)$ & $-\frac{1}{2} s_{2}$ & $\frac{1}{2 \sqrt{3}} s_{2}$ & $\frac{1}{2} s_{2}$ & & \\
\hline$T_{b}^{+}$ & $\begin{array}{c}T_{c}^{0} \pi^{+} \\
s_{1}+s_{4}\end{array}$ & & & & & \\
\hline \multirow[t]{2}{*}{$N_{b}^{0}$} & $T_{c}^{-} K^{+}$ & $T_{c}^{0} K^{0}$ & $N_{c}^{-} \pi^{+}$ & $N_{c}^{0} \pi^{0}$ & $N_{c}^{0} \eta$ & $\Theta_{c}^{0} \bar{K}^{0}$ \\
\hline & $\frac{1}{2}\left(s_{2}+s_{4}\right)$ & $\frac{1}{\sqrt{2}} s_{2}$ & $\frac{1}{2}\left(2 s_{1}+s_{3}\right)$ & $-\frac{1}{2 \sqrt{2}}\left(s_{3}-s_{4}\right)$ & $-\frac{1}{\sqrt{6}}\left(s_{2}-s_{3}-s_{4}\right)$ & $\frac{1}{\sqrt{2}} s_{3}$ \\
\hline \multirow[t]{2}{*}{$N_{b}^{+}$} & $T_{c}^{0} K^{+}$ & $N_{c}^{0} \pi^{+}$ & & & & \\
\hline & $\frac{1}{\sqrt{2}} s_{4}$ & $\frac{1}{2}\left(2 s_{1}+s_{4}\right)$ & & & & \\
\hline \multirow[t]{2}{*}{$\Theta_{b}^{+}$} & $N_{c}^{0} K^{+}$ & $\Theta_{c}^{0} \pi^{+}$ & & & & \\
\hline & $\frac{1}{\sqrt{2}} s_{4}$ & $s_{1}$ & & & & \\
\hline
\end{tabular}

$$
\begin{aligned}
\Gamma\left(T_{b}^{-} \rightarrow T_{c}^{0} \pi^{-}\right) & =2 \Gamma\left(T_{b}^{-} \rightarrow N_{c}^{0} K^{-}\right) \\
& =4 \Gamma\left(T_{b}^{0} \rightarrow T_{c}^{0} \pi^{0}\right)=12 \Gamma\left(T_{b}^{0} \rightarrow T_{c}^{0} \eta\right) \\
& =4 \Gamma\left(T_{b}^{0} \rightarrow N_{c}^{0} \bar{K}^{0}\right)=2 \Gamma\left(N_{b}^{0} \rightarrow T_{c}^{0} K^{0}\right) \\
\Gamma\left(N_{b}^{+} \rightarrow T_{c}^{0} K^{+}\right) & =\Gamma\left(\Theta_{b}^{+} \rightarrow N_{c}^{0} K^{+}\right) .
\end{aligned}
$$

More relations can be read off from the tables. These relations can be used to study the properties of heavy pen- taquarks and test the model provided that the decays have substantial branching ratios which require knowledge about the size of the $S U(3)_{f}$ invariant amplitudes.

Theoretical calculations of the decay amplitudes are very difficult since multiquarks are involved. However, for certain decays, the structure is very simple and can be related to experimentally measured modes, for example, $\Theta_{b}^{+} \rightarrow \Theta_{c}^{0} \pi^{+}$ can be related to $\Lambda_{b} \rightarrow \Lambda_{c}^{+} \pi^{-}$.

In $\Theta_{b}^{+} \rightarrow \Theta_{c}^{0} \pi^{+}$decay, the main contributions is due to

TABLE V. $S U(3)$ decay amplitudes for $S_{b} \rightarrow S_{c} \Pi$ with $\Delta S=1$. The Lorentz structure is similar to that in Table III.

\begin{tabular}{ccccccc}
\hline \hline$T_{b}^{-}$ & $T_{c}^{--} K^{+}$ & $T_{c}^{-} K^{0}$ & $N_{c}^{-} \pi^{0}$ & $N_{c}^{-} \eta$ & $N_{c}^{-} \pi^{-}$ & $\Theta_{c}^{0} K^{-}$ \\
\hline & $s_{1}+s_{3}$ & $\frac{1}{\sqrt{2}} s_{3}$ & $\frac{1}{2}\left(s_{2}+s_{4}\right)$ & $\frac{1}{2 \sqrt{3}}\left(s_{2}-s_{3}+s_{4}\right)$ & $\frac{1}{\sqrt{2}} s_{2}$ & $s_{2}$ \\
$T_{b}^{0}$ & $T_{c}^{-} K^{+}$ & $N_{c}^{-} \pi^{+}$ & $N_{c}^{0} \pi^{0}$ & $N_{c}^{0} \eta$ & $\Theta_{c}^{0} \bar{K}^{0}$ & \\
& $\frac{1}{2} s_{1}$ & $\frac{1}{2}\left(s_{2}+s_{4}\right)$ & $-\frac{1}{2 \sqrt{2}} s_{2}$ & $\frac{1}{2 \sqrt{6}} s_{2}$ & $\frac{1}{\sqrt{2}} s_{2}$ & \\
$T_{b}^{+}$ & $T_{c}^{0} K^{+}$ & $N_{c}^{0} \pi^{+}$ & & & \\
& $s_{1}$ & $\frac{1}{\sqrt{2}} s_{4}$ & & & \\
$N_{b}^{0}$ & $N_{c}^{-} K^{+}$ & $N_{c}^{0} K^{0}$ & $\Theta_{c}^{0} \pi^{0}$ & $\Theta_{c}^{0} \eta$ & \\
& $\frac{1}{2}\left(2 s_{1}+s_{2}+s_{3}+s_{4}\right)$ & $\frac{1}{2}\left(s_{2}+s_{3}\right)$ & $\frac{1}{2} s_{4}$ & $-\frac{1}{\sqrt{3}}\left(s_{2}+s_{3}-s_{4}\right)$ & \\
$N_{b}^{+}$ & $N_{c}^{0} K^{+}$ & $\Theta_{c}^{0} \pi^{+}$ & & & \\
& $\frac{1}{2}\left(2 s_{1}+s_{4}\right)$ & $\frac{1}{\sqrt{2}} s_{4}$ & & & \\
$\Theta_{b}^{+}$ & $\Theta_{c}^{0} K^{+}$ & $s_{1}+s_{4}$ & & & & \\
& & & & \\
\hline \hline
\end{tabular}


TABLE VI. $S U(3)$ decay amplitudes for $S_{b} \rightarrow S_{c} D$. The Lorentz structure is similar to that in Table III. For $S_{b} \rightarrow S_{c} D^{*}$, the Lorentz structure of the bi-spinor product is of the form $\bar{S}_{c} \gamma_{\mu}\left(1+b \gamma_{5}\right) S_{b}$.

\begin{tabular}{|c|c|c|}
\hline$\Delta S=0$ & & $\Delta S=1$ \\
\hline$U_{b}^{+}$ & $r_{31} U_{c}^{0} D_{d}$ & $r_{31} U_{c}^{0} D_{s}$ \\
\hline$D_{b}^{0}$ & $\left(r_{31}+r_{32}\right) D_{c}^{-} D_{d}+r_{32} U_{c}^{0} D_{u}+r_{32} S_{c}^{-} D_{s}$ & $r_{31} D_{c}^{-} D_{s}$ \\
\hline$S_{b}^{0}$ & $r_{31} S_{c}^{-} D_{d}$ & $\left(r_{31}+r_{32}\right) S_{c}^{-} D_{s}+r_{32} U_{c}^{0} D_{u}+r_{31} D_{c}^{-} D_{d}$ \\
\hline$U_{b}^{+}$ & $r T_{c}^{0} D_{s}-\frac{1}{\sqrt{2}} r N_{c}^{0} D_{d}$ & $\frac{1}{\sqrt{2}} r N_{c}^{0} D_{s}-r \Theta_{c}^{0} D_{d}$ \\
\hline$D_{b}^{0}$ & $\frac{1}{\sqrt{2}} r N_{c}^{0} D_{u}-\frac{1}{\sqrt{2}} r T_{c}^{-} D_{s}$ & $-\frac{1}{\sqrt{2}} r N_{c}^{-} D_{s}+r \Theta_{c}^{0} D_{u}$ \\
\hline$S_{b}^{0}$ & $\frac{1}{\sqrt{2}} r T_{c}^{-} D_{d}-r T_{c}^{0} D_{u}$ & $\frac{1}{\sqrt{2}} r N_{c}^{-} D_{d}-\frac{1}{\sqrt{2}} r N_{c}^{0} D_{u}$ \\
\hline$T_{b}^{-}$ & $s_{31} T_{c}^{--} D_{d}+\frac{1}{\sqrt{2}} s_{32} T_{c}^{-} D_{u}$ & $s_{31} T_{c}^{--} D_{s}+\frac{1}{\sqrt{2}} s_{32} N_{c}^{-} D_{u}$ \\
\hline$T_{b}^{0}$ & $\left(s_{31}+\frac{1}{2} s_{32}\right) T_{c}^{-} D_{d}+\frac{1}{\sqrt{2}} s_{32} T_{c}^{0} D_{u}$ & $s_{31} T_{c}^{-} D_{s}+\frac{1}{2} s_{32} N_{c}^{0} D_{u}+\frac{1}{2} s_{32} N_{c}^{-} D_{d}$ \\
\hline$T_{b}^{+}$ & $\left(s_{31}+s_{32}\right) T_{c}^{0} D_{d}$ & $s_{31} T_{c}^{0} D_{s}+\frac{1}{\sqrt{2}} s_{32} N_{c}^{0} D_{d}$ \\
\hline$N_{b}^{0}$ & $s_{31} N_{c}^{-} D_{d}+\frac{1}{2} s_{32} N_{c}^{0} D_{u}+\frac{1}{2} s_{32} T_{c}^{-} D_{s}$ & $\left(s_{31}+\frac{1}{2} s_{32}\right) N_{c}^{-} D_{s}+\frac{1}{\sqrt{2}} s_{32} N_{c}^{0} D_{u}$ \\
\hline$N_{b}^{+}$ & $\left(s_{31}+\frac{1}{2} s_{32}\right) N_{c}^{0} D_{d}+\frac{1}{\sqrt{2}} s_{32} T_{c}^{0} D_{s}$ & $\left(s_{31}+\frac{1}{2} s_{32}\right) N_{c}^{0} D_{s}+\frac{1}{\sqrt{2}} s_{32} \Theta_{c}^{0} D_{d}$ \\
\hline$\Theta_{b}^{+}$ & $s_{31} \Theta_{c}^{0} D_{d}+\frac{1}{\sqrt{2}} s_{32} N_{c}^{0} D_{s}$ & $\left(s_{31}+s_{32}\right) \Theta_{c}^{0} D_{s}$ \\
\hline
\end{tabular}

the factorized matrix elements where $\pi$ is emitted from two light quarks in the effective Hamiltonian, the transition of $\Theta_{b}^{+}$to $\Theta_{c}^{0}$ is due to the transition of a $\bar{b}$ quark to a $\bar{c}$ quark, and the structure of the rest of the four light quarks in the pentaquarks is basically preserved. Based on this intuitive picture, Ref. [18] relates $\Theta_{b}^{+} \rightarrow \Theta_{c}^{0} \pi^{+}$to $\Lambda_{b} \rightarrow \Lambda_{c}^{+} \pi^{-}$using heavy quark effective theory, and concludes that the branching ratios for these two processes are similar. The decay rate $\Gamma\left(\Theta_{b}^{+} \rightarrow \Theta_{c}^{0} \pi^{+}\right)$is estimated to be about $2.5 \Gamma\left(B^{0}\right.$ $\rightarrow D^{-} \pi^{-}$). This prediction can be tested at future collider experiments.

From Table IV, we see that $\Theta_{b}^{+} \rightarrow \Theta_{c}^{0} \pi^{+}$is proportional to the $S U(3)_{f}$ invariant amplitude $s_{1}$, one therefore can use the estimate in Ref. [18] to obtain an estimate for $s_{1}$. The invariant amplitudes $s_{2,3,4}$ involve more complicated topology and are much harder to estimate. Although it is difficult to know all decay amplitudes, knowing $s_{1}$ one can make some useful predictions. The branching ratios for processes in Tables IV and $\mathrm{V}$ which just depend on $s_{1}$ are therefore known. Up to mass splitting corrections in phase space, we obtain

$$
\begin{aligned}
& \Gamma\left(T_{b}^{0} \rightarrow T_{c}^{-} K^{+}\right) \sim \frac{1}{4} \frac{\left|V_{u s}\right|^{2}}{\left|V_{u d}\right|^{2}} \Gamma\left(\Theta_{b}^{+} \rightarrow \Theta_{c}^{0} \pi^{+}\right), \\
& \Gamma\left(T_{b}^{+} \rightarrow T_{c}^{0} K^{+}\right) \sim \frac{\left|V_{u s}\right|^{2}}{\left|V_{u d}\right|^{2}} \Gamma\left(\Theta_{b}^{+} \rightarrow \Theta_{c}^{0} \pi^{+}\right) .
\end{aligned}
$$

Using the heavy quark effective theory, one can also relate several other $S U(3)_{f}$ invariant amplitudes to $\Lambda_{b}$ $\rightarrow \Lambda_{c} \pi$ by realizing the fact that all amplitudes for the heavy pentaquark transitions of the form $R_{b, i} R_{c}^{i}, S_{b, i j} S_{c}^{i j}$ have similar factorization structure, and their strength should also be similar. We therefore expect that $s_{1} \sim r_{81} \sim r_{31} \sim s_{31}$.

One then has, up to mass splitting corrections in phase space, the following relations: 
TABLE VII. $S U(3)$ decay amplitudes for $B \rightarrow N \bar{R}_{c}\left(\bar{S}_{c}\right)$. The Lorentz structure should be understood to be $\bar{N}\left(1+b \gamma_{5}\right) R_{c}\left(S_{c}\right)$ since weak interaction can have $S$ - and $P$-wave amplitudes.

\begin{tabular}{|c|c|c|c|}
\hline$B_{u}$ decay & $\Delta S=0$ & & $\Delta S=-1$ \\
\hline $\bar{U}_{c}^{0} \Sigma^{-}$ & $\tilde{r}_{81}+\tilde{r}_{83}$ & $\bar{U}_{c}^{0} \Xi-$ & $\tilde{r}_{81}+\tilde{r}_{83}$ \\
\hline $\bar{T}_{c}^{0} \Xi^{-}$ & $\tilde{s}_{81}-\tilde{s}_{82}$ & $\bar{N}_{c}^{0} \Xi^{-}$ & $\frac{1}{\sqrt{2}}\left(\tilde{s}_{81}-\tilde{s}_{82}\right)$ \\
\hline $\bar{N}_{c}^{0} \Sigma^{-}$ & $-\frac{1}{\sqrt{2}}\left(\tilde{s}_{81}-\tilde{s}_{82}\right)$ & $\bar{\Theta}_{c}^{0} \Sigma^{-}$ & $-\left(\tilde{s}_{81}-\tilde{s}_{82}\right)$ \\
\hline \multicolumn{4}{|l|}{$B_{d}$ decay } \\
\hline $\bar{U}_{c}^{0} \Sigma^{0}$ & $-\frac{1}{\sqrt{2}}\left(\tilde{r}_{81}-\tilde{r}_{82}\right)$ & $\bar{U}_{c}^{0} \Xi^{0}$ & $\tilde{r}_{81}$ \\
\hline $\bar{U}_{c}^{0} \Lambda$ & $\frac{1}{\sqrt{6}}\left(\tilde{r}_{81}+\tilde{r}_{82}\right)$ & $\bar{D}_{c}^{-} \Xi \Xi^{-}$ & $\tilde{r}_{83}$ \\
\hline $\bar{D}_{c}^{-} \Sigma^{-}$ & $\tilde{r}_{82}+\tilde{r}_{83}$ & $\bar{N}_{c}^{-} \Xi^{-}$ & $-\frac{1}{\sqrt{2}} \tilde{s}_{81}$ \\
\hline $\bar{S}_{c}^{-} \Xi^{-}$ & $\tilde{r}_{82}$ & $\bar{N}_{c}^{0} \Xi^{0}$ & $-\frac{1}{\sqrt{2}} \tilde{s}_{82}$ \\
\hline $\bar{T}_{c}^{-} \Xi^{-}$ & $-\frac{1}{\sqrt{2}}\left(\tilde{s}_{81}+\tilde{s}_{83}\right)$ & $\bar{\Theta}_{c}^{0} \Sigma^{0}$ & $\frac{1}{\sqrt{2}}\left(\tilde{s}_{81}-\tilde{s}_{82}\right)$ \\
\hline $\bar{T}_{c}^{0} \Xi^{0}$ & $-\left(\tilde{s}_{82}+\tilde{s}_{83}\right)$ & $\bar{\Theta}_{c}^{0} \Lambda$ & $\frac{1}{\sqrt{6}}\left(\tilde{s}_{81}+\tilde{s}_{82}\right)$ \\
\hline $\bar{N}_{c}^{-} \Sigma^{-}$ & $\frac{1}{\sqrt{2}} \tilde{s}_{83}$ & & \\
\hline $\bar{N}_{c}^{0} \Sigma^{0}$ & $\frac{1}{2}\left(\tilde{s}_{81}-\tilde{s}_{82}-\tilde{s}_{83}\right)$ & & \\
\hline $\bar{N}_{c}^{0} \Lambda$ & $\frac{1}{2 \sqrt{3}}\left(\tilde{s}_{81}+\tilde{s}_{82}+3 \tilde{s}_{83}\right)$ & & \\
\hline $\bar{\Theta}_{c}^{0} n$ & $\tilde{s}_{83}$ & & \\
\hline \multicolumn{4}{|l|}{$B_{s}$ decay } \\
\hline $\bar{U}_{c}^{0} n$ & $\tilde{r}_{81}$ & $\bar{U}_{c}^{0} \Sigma^{0}$ & $\frac{1}{\sqrt{2}} \tilde{r}_{82}$ \\
\hline $\bar{S}_{c}^{-} \Sigma^{-}$ & $r_{83}$ & $\bar{U}_{c}^{0} \Lambda$ & $-\frac{1}{\sqrt{6}}\left(2 \tilde{r}_{81}-\tilde{r}_{82}\right)$ \\
\hline $\bar{T}_{c}^{-} \Sigma^{-}$ & $\frac{1}{\sqrt{2}} \tilde{s}_{81}$ & $\bar{D}_{c}^{-} \Sigma^{-}$ & $\tilde{r}_{82}$ \\
\hline $\bar{T}_{c}^{0} \Sigma^{0}$ & $-\frac{1}{\sqrt{2}} \tilde{s}_{81}$ & $\bar{S}_{c}^{-} \Xi^{-}$ & $\tilde{r}_{82}+\tilde{r}_{83}$ \\
\hline $\bar{T}_{c}^{0} \Lambda$ & $-\frac{1}{\sqrt{6}}\left(\tilde{s}_{81}-2 \tilde{s}_{82}\right)$ & $\bar{T}_{c}^{-} \Xi{ }^{-}$ & $-\frac{1}{\sqrt{2}} \tilde{s}_{83}$ \\
\hline \multirow[t]{5}{*}{$\bar{N}_{c}^{0} n$} & $\frac{1}{\sqrt{2}} \tilde{s}_{82}$ & $\bar{T}_{c}^{0} \Xi^{0}$ & $-\tilde{s}_{83}$ \\
\hline & & $\bar{N}_{c}^{-} \Sigma^{-}$ & $\frac{1}{\sqrt{2}}\left(\tilde{s}_{81}+\tilde{s}_{83}\right)$ \\
\hline & & $\bar{N}_{c}^{0} \Sigma^{0}$ & $-\frac{1}{2}\left(\tilde{s}_{81}+\tilde{s}_{83}\right)$ \\
\hline & & $\bar{N}_{c}^{0} \Lambda$ & $-\frac{1}{2 \sqrt{3}}\left(\tilde{s}_{81}-2 \tilde{s}_{82}-3 \tilde{s}_{83}\right)$ \\
\hline & & $\bar{\Theta}_{c}^{0} n$ & $\tilde{s}_{82}+\tilde{s}_{83}$ \\
\hline
\end{tabular}

TABLE VIII. $S U(3)$ decay amplitudes for $B \rightarrow \bar{N}\left(R_{c}, S_{c}\right)$ with $\Delta S=0$. The Lorentz structure is of the form $\bar{R}_{c}\left(\bar{S}_{c}\right)\left(1+b \gamma_{5}\right) N$.

\begin{tabular}{|c|c|c|}
\hline$B_{u}$ & $\Sigma^{+} U_{c}^{0}$ & $\left(\tilde{r}_{31}+\tilde{r}_{32}+\tilde{r}_{61}+\tilde{r}_{62}\right)$ \\
\hline & $\Sigma^{0} D_{c}^{-}$ & $-\frac{1}{\sqrt{2}}\left(\tilde{r}_{31}+\tilde{r}_{32}-\tilde{r}_{61}+\tilde{r}_{62}\right)$ \\
\hline & $\bar{\Lambda} D_{c}^{-}$ & $-\frac{1}{\sqrt{6}}\left(\tilde{r}_{31}-\tilde{r}_{32}+2 \tilde{r}_{33}-\tilde{r}_{61}-\tilde{r}_{62}\right)$ \\
\hline & $\Xi^{0} S_{c}^{-}$ & $\tilde{r}_{32}-\tilde{r}_{33}+\tilde{r}_{62}$ \\
\hline & $\Xi^{-} T_{c}^{--}$ & $\tilde{s}_{31}-\tilde{s}_{61}-\tilde{s}_{62}$ \\
\hline & $\Xi^{0} T_{c}^{-}$ & $\frac{1}{\sqrt{2}}\left(\tilde{s}_{31}+\tilde{s}_{61}-\tilde{s}_{62}\right)$ \\
\hline & $\Sigma^{0} N_{c}^{-}$ & $\frac{1}{2}\left(\tilde{s}_{32}+2 \tilde{s}_{61}+\tilde{s}_{62}\right)$ \\
\hline & $\bar{\Lambda} N_{c}^{-}$ & $-\frac{1}{2 \sqrt{3}}\left(2 \tilde{s}_{31}-\tilde{s}_{32}-3 \tilde{s}_{62}\right)$ \\
\hline & $\Sigma^{+} N_{c}^{0}$ & $\frac{1}{\sqrt{2}}\left(\tilde{s}_{32}+\tilde{s}_{62}\right)$ \\
\hline & $\bar{p} \Theta_{c}^{0}$ & $\tilde{s}_{32}+\tilde{s}_{62}$ \\
\hline \multirow[t]{10}{*}{$B_{d}$} & $\Sigma^{0} U_{c}^{0}$ & $-\frac{1}{\sqrt{2}}\left(\tilde{r}_{31}+\tilde{r}_{32}+\tilde{r}_{61}-\tilde{r}_{62}\right)$ \\
\hline & $\bar{\Lambda} U_{c}^{0}$ & $\frac{1}{\sqrt{6}}\left(\tilde{r}_{31}-\tilde{r}_{32}+2 \tilde{r}_{33}+\tilde{r}_{61}+\tilde{r}_{62}\right)$ \\
\hline & $\Sigma^{-} D_{c}^{-}$ & $-\left(\tilde{r}_{31}+\tilde{r}_{32}-\tilde{r}_{61}-\tilde{r}_{62}\right)$ \\
\hline & $\Xi^{-} S_{c}^{-}$ & $-\left(\tilde{r}_{32}-\tilde{r}_{33}+\tilde{r}_{62}\right)$ \\
\hline & $\Xi^{-} T_{c}^{--}$ & $\frac{1}{\sqrt{2}}\left(\tilde{s}_{31}-\tilde{s}_{61}+\tilde{s}_{62}\right)$ \\
\hline & $\Xi^{0} T_{c}^{0}$ & $\tilde{s}_{31}+\tilde{s}_{61}+\tilde{s}_{62}$ \\
\hline & $\Sigma^{-} N_{c}^{-}$ & $\frac{1}{\sqrt{2}}\left(\tilde{s}_{32}-\tilde{s}_{62}\right)$ \\
\hline & $\bar{\Lambda} N_{c}^{0}$ & $-\frac{1}{2 \sqrt{3}}\left(2 \tilde{s}_{31}-\tilde{s}_{32}+3 \tilde{s}_{62}\right)$ \\
\hline & $\bar{\Sigma}^{0} N_{c}^{0}$ & $-\frac{1}{2}\left(\tilde{s}_{32}-2 \tilde{s}_{61}-\tilde{s}_{62}\right)$ \\
\hline & $\bar{n} \Theta_{c}^{0}$ & $\tilde{s}_{32}-\tilde{s}_{62}$ \\
\hline \multirow[t]{6}{*}{$B_{s}$} & $\Xi^{0} U_{c}^{0}$ & $\tilde{r}_{31}+\tilde{r}_{33}+\tilde{r}_{61}$ \\
\hline & $\Xi^{-} D_{c}^{-}$ & $-\left(\tilde{r}_{31}+\tilde{r}_{33}-\tilde{r}_{61}\right)$ \\
\hline & $\Xi^{-} N_{c}^{-}$ & $\frac{1}{\sqrt{2}}\left(\tilde{s}_{31}+\tilde{s}_{32}-\tilde{s}_{61}\right)$ \\
\hline & $\Xi^{0} N_{c}^{0}$ & $\frac{1}{\sqrt{2}}\left(\tilde{s}_{31}+\tilde{s}_{32}+\tilde{s}_{61}\right)$ \\
\hline & $\Sigma^{0} \Theta_{c}^{0}$ & $\sqrt{2} \tilde{s}_{61}$ \\
\hline & $\bar{\Lambda} \Theta_{c}^{0}$ & $-\sqrt{\frac{2}{3}}\left(\tilde{s}_{31}+\tilde{s}_{32}\right)$ \\
\hline
\end{tabular}


TABLE IX. $S U(3)$ decay amplitudes for $B \rightarrow \bar{N}\left(R_{c}, S_{c}\right)$ with $\Delta S=-1$. The Lorentz structure is the same as Table VIII.

\begin{tabular}{|c|c|c|}
\hline$B_{u}$ & $\bar{p} U_{c}^{0}$ & $\tilde{r}_{31}+\tilde{r}_{32}+\tilde{r}_{61}+\tilde{r}_{62}$ \\
\hline & $\bar{n} D_{c}^{-}$ & $\tilde{r}_{32}-\tilde{r}_{33}+\tilde{r}_{62}$ \\
\hline & $\Sigma^{0} S_{c}^{-}$ & $-\frac{1}{\sqrt{2}}\left(\tilde{r}_{31}+\tilde{r}_{33}-\tilde{r}_{61}\right)$ \\
\hline & $\bar{\Lambda} S_{c}^{-}$ & $-\frac{1}{\sqrt{6}}\left(\tilde{r}_{31}+2 \tilde{r}_{32}-\tilde{r}_{33}-\tilde{r}_{61}+2 \tilde{r}_{62}\right)$ \\
\hline & $\bar{\Sigma}^{-} T_{c}^{--}$ & $-\left(\tilde{s}_{31}-\tilde{s}_{61}-\tilde{s}_{62}\right)$ \\
\hline & $\Sigma^{0} T_{c}^{-}$ & $-\frac{1}{2}\left(\tilde{s}_{31}-\tilde{s}_{32}-\tilde{s}_{61}-2 \tilde{s}_{62}\right)$ \\
\hline & $\bar{\Lambda} T_{c}^{-}$ & $-\frac{1}{2 \sqrt{3}}\left(\tilde{s}_{31}+\tilde{s}_{32}+3 \tilde{s}_{61}\right)$ \\
\hline & $\bar{\Sigma}^{+} T_{c}^{0}$ & $-\left(\tilde{s}_{32}+\tilde{s}_{62}\right)$ \\
\hline & $\bar{n} N_{c}^{-}$ & $-\frac{1}{\sqrt{2}}\left(\tilde{s}_{31}+\tilde{s}_{61}-\tilde{s}_{62}\right)$ \\
\hline & $\bar{p} N_{c}^{0}$ & $-\frac{1}{\sqrt{2}}\left(\tilde{s}_{32}+\tilde{s}_{62}\right)$ \\
\hline \multirow[t]{6}{*}{$B_{d}$} & $\bar{n} U_{c}^{0}$ & $\left(\tilde{r}_{31}+\tilde{r}_{33}+\tilde{r}_{61}\right)$ \\
\hline & $\Sigma^{-} S_{c}^{-}$ & $-\left(\tilde{r}_{31}+\tilde{r}_{33}-\tilde{r}_{61}\right)$ \\
\hline & $\bar{\Sigma}^{-} T_{c}^{-}$ & $-\frac{1}{\sqrt{2}}\left(\tilde{s}_{31}+\tilde{s}_{32}-\tilde{s}_{61}\right)$ \\
\hline & $\bar{\Sigma}^{0} T_{c}^{0}$ & $\frac{1}{\sqrt{2}}\left(\tilde{s}_{31}+\tilde{s}_{32}-\tilde{s}_{61}\right)$ \\
\hline & $\bar{\Lambda} T_{c}^{0}$ & $-\frac{1}{\sqrt{6}}\left(\tilde{s}_{31}+\tilde{s}_{32}+3 \tilde{s}_{61}\right)$ \\
\hline & $\bar{n} N_{c}^{0}$ & $-\frac{1}{\sqrt{2}}\left(\tilde{s}_{31}+\tilde{s}_{32}+\tilde{s}_{61}\right)$ \\
\hline \multirow[t]{10}{*}{$B_{s}$} & $\Sigma^{0} U_{c}^{0}$ & $-\frac{1}{\sqrt{2}}\left(\tilde{r}_{32}-\tilde{r}_{33}-\tilde{r}_{62}\right)$ \\
\hline & $\bar{\Lambda} U_{c}^{0}$ & $-\frac{1}{\sqrt{6}}\left(2 \tilde{r}_{31}+\tilde{r}_{32}+r_{33}+2 \tilde{r}_{61}-\tilde{r}_{62}\right)$ \\
\hline & $\Sigma^{-} D_{c}^{-}$ & $-\left(\tilde{r}_{32}-\tilde{r}_{33}-\tilde{r}_{62}\right)$ \\
\hline & $\Xi^{-} S_{c}^{-}$ & $-\left(\tilde{r}_{31}+\tilde{r}_{32}-\tilde{r}_{61}-\tilde{r}_{62}\right)$ \\
\hline & $\Xi^{-} T_{c}^{-}$ & $-\frac{1}{\sqrt{2}}\left(\tilde{s}_{32}-\tilde{s}_{62}\right)$ \\
\hline & $\Xi^{0} T_{c}^{0}$ & $-\left(\tilde{s}_{32}-\tilde{s}_{62}\right)$ \\
\hline & $\Sigma^{0} N_{c}^{0}$ & $\frac{1}{2}\left(\tilde{s}_{31}-\tilde{s}_{61}+\tilde{s}_{62}\right)$ \\
\hline & $\bar{\Lambda} N_{c}^{0}$ & $-\frac{1}{2 \sqrt{3}}\left(\tilde{s}_{31}-2 \tilde{s}_{32}+3 \tilde{s}_{61}+3 \tilde{s}_{62}\right)$ \\
\hline & $\bar{\Sigma}^{-} N_{c}^{-}$ & $-\frac{1}{\sqrt{2}}\left(\tilde{s}_{31}-\tilde{s}_{61}+\tilde{s}_{62}\right)$ \\
\hline & $\overline{n \Theta_{c}^{0}}$ & $-\left(\tilde{s}_{31}+\tilde{s}_{61}+\tilde{s}_{62}\right)$ \\
\hline
\end{tabular}

$$
\begin{aligned}
& \Gamma\left(\Theta_{b}^{+} \rightarrow \Theta_{c}^{0} \pi^{+}\right) \sim \Gamma\left(S_{b}^{0} \rightarrow S_{c}^{-} \pi^{+}\right) \sim \frac{\left|V_{u d}\right|^{2}}{\left|V_{u s}\right|^{2}} \Gamma\left(D_{b}^{0} \rightarrow D_{0}^{-} K^{+}\right) \\
& \sim \frac{\left|V_{u d}\right|^{2}}{\left|V_{c d}\right|^{2}}\left[\Gamma\left(U_{b}^{+} \rightarrow U_{c}^{0} D_{d}\right),\right. \\
&\left.\Gamma\left(S_{b}^{0} \rightarrow S_{c}^{-} D_{d}\right)\right] \sim \frac{\left|V_{u d}\right|^{2}}{\left|V_{c s}\right|^{2}}\left[\Gamma\left(U_{b}^{+} \rightarrow U_{c}^{0} D_{s}\right),\right. \\
& \Gamma\left(U_{b}^{+}\right.\left.\left.\rightarrow D_{c}^{-} D_{s}\right), \quad \Gamma\left(S_{b}^{0} \rightarrow D_{c}^{-} D_{d}\right)\right] \\
& \sim \frac{\left|V_{u d}\right|^{2}}{\left|V_{c d}\right|^{2}}\left[\Gamma\left(T_{b}^{-} \rightarrow T_{c}^{--} D_{d}\right),\right. \\
&\left.\Gamma\left(N_{b}^{0} \rightarrow N_{c}^{-} D_{d}\right), \quad \Gamma\left(\Theta_{b}^{+} \rightarrow \Theta_{c}^{0} D_{d}\right)\right] \\
&\left.\Gamma\left(T_{b}^{0} \rightarrow T_{c}^{-} D_{s}\right), \quad \Gamma\left(T_{b}^{+} \rightarrow T_{c}^{0} D_{s}\right)\right] . \\
& \sim \frac{\left|V_{u d}\right|^{2}}{\left|V_{c s}\right|^{2}}\left[\Gamma\left(T_{b}^{-} \rightarrow T_{c}^{--} D_{s}\right),\right. \\
&
\end{aligned}
$$

The above relations also hold for processes with replacing $D$ by $D^{*}$.

Pentaquark properties can also be studied at $B$ factories. We have studied $B \rightarrow N \bar{R}_{c}\left(\bar{S}_{c}\right)$ and $B \rightarrow \bar{N} R_{c}\left(S_{c}\right)$ decays. From Tables VII, VIII, and IX, we see that there are several relations. For example,

$$
\begin{gathered}
\Gamma\left(B_{u} \rightarrow \bar{U}_{c}^{0} \Sigma^{-}\right)=\frac{\left|V_{u d}\right|^{2}}{\left|V_{u s}\right|^{2}} \Gamma\left(B_{u} \rightarrow \bar{U}_{c} \Xi^{-}\right), \\
\Gamma\left(B_{u} \rightarrow \bar{p} U_{c}^{0}\right)=\frac{\left|V_{u d}\right|^{2}}{\left|V_{u s}\right|^{2}} \Gamma\left(B_{u} \rightarrow \bar{\Sigma}^{+} U_{c}^{0}\right) .
\end{gathered}
$$

Should the heavy pentaquarks be discovered, these relations can also provide important information. The decay amplitudes for $B \rightarrow N \bar{R}_{c}\left(\bar{S}_{c}\right)$ and $B \rightarrow \bar{N} R_{c}\left(S_{c}\right)$ are difficult to estimate. We are not able to provide any reliable estimate, except that we expect them to be smaller than $B \rightarrow N \bar{\Lambda}_{c}$ amplitudes.

Using the same formulation, one can also study $B$ decays into an ordinary baryon $N$ and a light pentaquark, such as a $\Theta^{+}$in the antidecuplet. We, however, expect that the branching ratios are smaller than $B \rightarrow N \bar{N}$. Since $B \rightarrow N \bar{N}$ have small branching ratios, it may be difficult to study $B$ $\rightarrow N \bar{\Theta}^{+}$experimentally. This situation may change if one studies three body decays of $B$ with a light pentaquark in the final states, such as $B \rightarrow D p(n) \Theta$. The $B \rightarrow D K$ decay has a branching ratio of order a few times $10^{-4}$. The $K$ has a strong coupling to $p(n) \Theta^{+}$which can be determined from the $\Theta^{+}$decay. With $\Gamma_{\Theta}$ of $\Theta^{+}$being about one $\mathrm{MeV}$, one can obtain a branching ratio for $B \rightarrow D p(n) \Theta$ as large as $10^{-6}$ which is within the reach of near future $B$ factories. 


\section{ACKNOWLEDGMENTS}

We would like to thank H.-Y. Cheng and Shi-Lin Zhu for useful discussions. We thank H.-Y. Cheng for informing us of a paper by Cheng, Chua, and Hwang [20] where some of the heavy pentaquark decays discussed in this paper are also considered before its publication. We also thank M.-L. Yan for bringing his early work on pentaquark to our attentions. This work is partially supported by Grants from NSC and NNSF.
[1] LEPS Collaboration, T. Nakano et al., Phys. Rev. Lett. 91, 012002 (2003); DIANA Collaboration, V.V. Barmin et al., Phys. Atom. Nucl. 66, 1715 (2003); CLAS Collaboration, S. Stepanyan et al., Phys. Rev. Lett. 91, 252001 (2003); SAPHIR Collaboration, J. Barth et al., hep-ex/0307083; NA49 Collaboration, C. Alt et al., Phys. Rev. Lett. 92, 042003 (2004); HERMES Collaboration, A. Airapetian et al., Phys. Lett. B 585, 213 (2004); COSY-TOF Collaboration, M. Abdel-Bary et al., hep-ph/0403011; SVD Collaboration, A. Aleev et al., hep-ex/0401024.

[2] BES Collaboration, J.Z. Bai et al., hep-ex/0404012; K. Knopflet, M. Zavertyaev, and T. Zivko, for the HERA-B Collaboration, hep-ex/0403020.

[3] A.V. Manohar, Nucl. Phys. B248, 19 (1984); M. Chemtob, ibid. B256, 600 (1985); M. Praszalowicz, in Skyrmions and Anomalies, edited by M. Jezabeck and M. Praszolowicz (World Scientific, Singapore, 1987), 112; Phys. Lett. B 575, 234 (2003); D. Diakonov, V. Petrov, and M.V. Polyakov, Z. Phys. A 359, 305 (1997).

[4] Y. Oh, Phys. Lett. B 331, 362 (1994); M.-L. Yan and X.-H. Meng, Commun. Theor. Phys. 24, 435 (1995); F. Stancu and D. Riska, Phys. Rev. Lett. 575, 242 (2003); hep-ph/0402044; M.-L. Yuan, X.-H.F. Huang, Z. Zhang, Y.-W. Yu, and B.-S. Zou, Phys. Lett. B 586, 69 (2004).

[5] R.L. Jaffe and F. Wilczek, Phys. Rev. Lett. 91, 232003 (2003).

[6] M. Kaliner and H.J. Lipkin, hep-ph/0307243; hep-ph/0307343; Phys. Lett. B 575, 249 (2003).

[7] F. Csikor, Z. Fodor, S.D. Katz, and T.G. Kovacs, J. High
Energy Phys. 11, 070 (2003); T.-W. Chiu and T.-H. Hsieh, hep-ph/0403020.

[8] S. Sasaki, hep-lat/0310014.

[9] S. Fleck, C. Gignoux, J. Richard, and B. Silvestre-Brac, Phys. Lett. B 220, 616 (1989); S. Gerasyuta and V. Kochkin, Int. J. Mod. Phys. E 12, 793 (2003); C. Carlson, C. Carone, H. Kwee, and V. Nazaryan, Phys. Lett. B 373, 101 (2003); S. Capstick, P. Page, and W. Roberts, ibid. 570, 185 (2003); R. Williams and P. Gueye, nucl-th/0308058; D.E. Kahana and S.H. Kahana, hep-ph/0310026; Y.-X. Liu, J.-S. Li, and C.-G. Bao, hep-ph/0401197.

[10] K. Cheung, hep-ph/0308176.

[11] C. Gignoux, B. Silvestre-Brac, and J. Richard, Phys. Lett. B 193, 323 (1987); H. Lpkin, ibid. 195, 484 (1987); E791 Collaboration, E.M. Aitala et al., Phys. Rev. Lett. 81, 44 (1998).

[12] Bin Wu and Bo-Qiang Ma, hep-ph/0402244.

[13] Shi-Lin Zhu, Phys. Rev. Lett. 91, 232002 (2003); J. Sugiyama, T. Doi, and M. Oka, Phys. Lett. B 581, 167 (2004); E. Shuryak and I. Zahed, hep-ph/0310270.

[14] M. Genovese, J.-M. Richard, F. Stancu, and S. Pepin, Phys. Lett. B 425, 171 (1998); F. Stancu, Phys. Rev. D 58, 111501 (1998).

[15] I. Stewart, M. Wessling, and M. Wise, hep-ph/0402076.

[16] P.-Z. Huang et al., hep-ph/0401191.

[17] J. Rosner, hep-ph/0312269; T. Browder, I. Klebanov, and D. Marlow, Phys. Lett. B 587, 62 (2004).

[18] A. Leibovich, Z. Ligeti, I. Stewart, and M. Wise, hep-ph/031219.

[19] H1 Collaboration, A. Aktas et al., hep-ex/0403017.

[20] H.-Y. Cheng, C.-K. Chua, and C.-W. Hwang, hep-ph/043232. 